\title{
A Global Perspective of Stenting After Ureteroscopy: An Observational Multicenter Cohort Study
}

\author{
Ranan Dasgupta, ${ }^{1}$ Teng Aik Ong, ${ }^{2}$ Jasmine Lim, ${ }^{2}$ Retnagowri Rajandram, ${ }^{2}$ Xiaofeng Gao, ${ }^{3}$ \\ Lukman Hakim, ${ }^{4}$ Patrick Mburugu, ${ }^{5}$ Rohit Ajmera, ${ }^{6}$ Emrah Yuruk, ${ }^{7}$ Yeong-Shiau Pu, ${ }^{8}$ \\ Petrisor Geavlete, ${ }^{9}$ Raed A. Azhar, ${ }^{10}$ Shingai Mutambirwa, ${ }^{11}$ Joyce Baard ${ }^{\otimes 12}$ \\ ${ }^{1}$ Imperial Urology, Imperial College Healthcare NHS Trust, Charing Cross Hospital, London, United Kingdom ${ }^{2}$ Department of Surgery, Faculty of Medicine, University of \\ Malaya, Kuala Lumpur, Malaysia ${ }^{3}$ Department of Urology, Changhai Hospital, Second Military Medical University, Shanghai, China ${ }^{4}$ Department of Urology, Airlangga \\ University, Dr Soetomo Hospital, Surabaya, Indonesia ${ }^{5}$ Department of Urology, Kenyatta National Hospital, Nairobi, Kenya ${ }^{6}$ Jawahar Lal Nehru (JLN) Medical College, \\ Rajasthan, India ${ }^{7}$ Department of Urology, Bağcılar Training and Research Hospital, İstanbul, Turkey ${ }^{8}$ Department of Urology, College of Medicine, National Taiwan \\ University, Taipei, Taiwan ${ }^{9}$ Department of Urology, Saint John Emergency Clinical Hospital, Bucharest, Romania ${ }^{10}$ Department of Urology, King Abdulaziz University, \\ Jeddah, Saudi Arabia ${ }^{11}$ Department of Nuclear Medicine, Sefako Makgatho University, Pretoria, South Africa ${ }^{12}$ Department of Urology, Amsterdam UMC, University of \\ Amsterdam, the Netherlands
}

\section{Abstract}

Objectives With an increasing number of patients undergoing ureteroscopic surgery worldwide for stone disease, and the concomitant pressures on health care resources, we aimed to review global patterns for ureteric stenting following ureteroscopy. With a centralized electronic database, a longitudinal cohort study was designed to help define the indications for stenting, type of drainage, and methods of stent removal.

Methods This multicenter study was conducted prospectively, with centralized data acquisition by uCARE (research arm of the Société Internationale d'Urologie), and registered at ClinicalTrials.gov (NCT03567421). Along with baseline demographic data, details were entered for stone imaging characteristics, intraoperative information, including urine culture, and exit strategy for stent removal. An independent audit was undertaken to sample the accuracy of data entered across the sites.

Results In total, 2544 patients were included from 50 centers in 15 countries. There were 1969 patients with ureteric stones, and 942 with renal stones. While $41 \%$ ureteric stones were distal (median length $10 \mathrm{~mm}$ ), $52 \%$ renal stones were in the lower pole (median diameter $11 \mathrm{~mm}$ ). The majority of patients $(68.8 \%)$ were given antibiotics at induction; $20.6 \%$ were "pre-stented" before the ureteroscopy, and a high percentage were stented following ureteroscopy (91\%). The majority of the stents (85.1\%) were polyurethane, mean duration of stent after surgery was 27 days, and $80 \%$ of stent removals were undertaken in the operating room.

Conclusion This is one of the largest prospective global cohort studies, reflecting widespread usage of stenting, despite emerging evidence to the contrary. Stent removals may also be modified with time, as the pressure on health care resources mounts.

\section{Introduction}

Globally, the past decade has witnessed the increasing trend towards ureterorenoscopic treatment of stone disease [1-3]. These procedures may involve use of semi-rigid or flexible instruments, and either digital or fiber optic technology. The miniaturization of the scopes, along with the availability of tools (laser fibers, guidewires, baskets), has led to ureterorenoscopy (URS) overtaking extracorporeal shockwave lithotripsy (ESWL) in terms of numbers of

\section{Key Words}

Ureteral stent, urolithiasis, ureteroscopy, stent
Competing Interests

None declared.

\section{Article Information}

Received on October 2, 2020

Accepted on December 1, 2020

Soc Int Urol J. 2021;2(2):96-105

DOI: https://10.48083/HRLS8587 


\author{
Abbreviations \\ ASA American Society of Anesthesiologists \\ ESWL Extracorporeal shockwave lithotripsy \\ IQR Interquartile range \\ PCNL Percutaneous nephrolithotomy \\ SIU Société Internationale d’Urologie \\ UAS Ureteral access sheath \\ URS Ureteroscopy ureterorenoscopy \\ UTI Urinary tract infection
}

procedures for upper tract stone disease[4]. It is accepted that patients will sometimes need a ureteric stent at the end of a URS procedure, although debate remains about the indications for this[5]. The use of a stent has associated morbidity and is often the aspect of a URS procedure that a patient remembers most vividly.

While different guidelines and reviews have attempted to offer broad outlines on the appropriate use of stents, there remains much variation in practice with respect to their usage. Data from 11885 URS procedures in the Clinical Research Office of the Endourological Society (CROES) study gave an insight into the frequency of use of stents ( $84.1 \%$ for all procedures, $60 \%$ after ureteric stone treatment, $80 \%$ after renal stone treatment), highlighting the need for further detail on the indications and features of these stent insertions [6].

The importance of this common aspect of stone treatment, with implications for health care costs and delivery worldwide, led to the current longitudinal cohort study with centralized independent data acquisition, allowing us to analyze the practice patterns across the globe and address the key questions of indication for stent insertion, the type and duration of drainage, and patterns for stent removal techniques.

\section{Methods}

This is a prospective multicenter observational longitudinal cohort study of patients with ureteric or renal stones treated by ureterorenoscopy. The study was executed by uCARE, the Office of Research of the Société Internationale d'Urologie (SIU).

To meet the inclusion criteria, patients had to be $\geq 18$ years old, able to give written consent, and suitable for treatment of a ureteric or renal stone by ureteroscopy. The estimated enrollment for this registry was at least 30 patients from 50 participating centers worldwide. All participating centers obtained ethical approval or a waiver from their local Institutional Review Board (IRB).
Patient data were anonymized, stored in a centralized database, and audited at the conclusion of the study period. This study was registered at ClinicalTrials.gov (NCT03567421) on June 25, 2018.

Study assessments: Data entry was undertaken at 3 timepoints: T1 (baseline, on the day of the URS), T2a (day of stent removal for stented patients) or T2b (first outpatient clinic, for unstented patients). The study scheme is outlined in Figure 1. The data collected were

Baseline characteristics: age, gender, American Society of Anesthesiologists physical status classification system (ASA I-IV), body mass index, urinary tract infection (UTI), prior stent use, previous stone treatment, and other morbidity.

Stone characteristics: location, number of stones, largest dimension (stone burden, surface area $=$ length $\times$ width $\times 0.25 \times 3.14159$ ).

\section{FIGURE 1.}

Study scheme

\section{Patient Selection}

1. Aged $\geq 18$ years old with a ureter or renal stone 2. Plan for ureteroscopic (URS) treatment

\section{Day of Surgery (T1)}

Data collected: baseline characteristics, stone characteristics, intraoperative data and exit strategy (stending status)

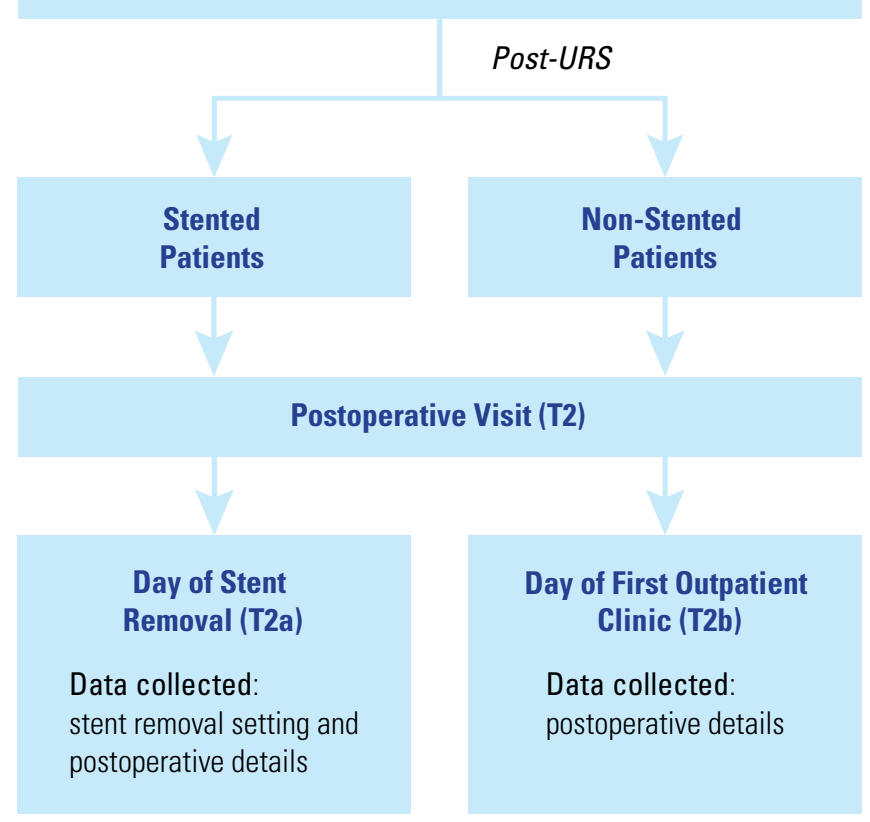


Intraoperative information: use of antibiotics, use of anesthesia, whether pre-stented (and indication for pre-stenting), need for ureteric dilatation, whether semi-rigid and/or flexible ureteroscopy, use of ureteral access sheath (UAS), type of lithotripsy energy, duration of surgery (defined as from passage of transurethral instruments to insertion of stent or ureteric drainage catheter), stone-free status assessed endoscopically.

Exit strategy: ureteric drainage (stent or ureter catheter), type of stent, indication, and indwelling time.

Stent removal: actual indwelling time, type of anesthesia for stent removal.

Data are presented as descriptive information, reporting mean (with standard deviation) or median (interquartile range) for normally distributed and skewed (continuous) variables, respectively. Dichotomous or categorial numbers are reported as absolute numbers or as percentages.

\section{Results}

The study was launched on June 1, 2018, and data collected until end of August 2019. A total of 2544 patients were included from 50 centers from 15 countries (Figure 2 and Appendix 1 for an overview of participating centers).

\section{FIGURE 2.}

Inclusion per country

\begin{tabular}{c|c} 
South Korea & 3 \\
South Africa & 5 \\
United States & 8
\end{tabular}

$\begin{array}{rr}\text { Iraq } & 41 \\ \text { Greece } & 42\end{array}$

Saudi Arabia $\quad 48$
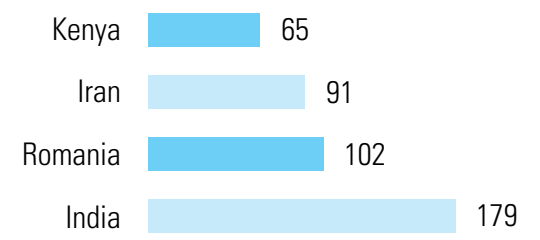

Indonesia

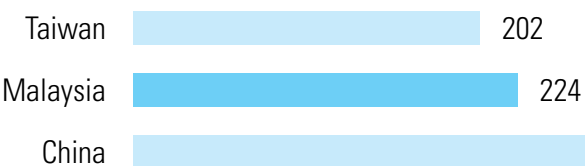

\section{Baseline Characteristics}

Of a total of 2544 patients, 2540 with complete case records were included. Baseline characteristics at inclusion are described in Table 1.

Our cohort had a mean age of 49.0 years (range 18 to 93 years), $64.3 \%$ were male, and the majority had an ASA classification 1 (51.5\%) or ASA 2 (40.4\%). Diabetes mellitus (13.9\%) and previous urinary tract infection $(16 \%)$ were frequently reported comorbidities.

Of the 671 patients (26.4\% of total) who had undergone previous stone surgery, $362(53.9 \%)$ had experience of previous ureteroscopy, 272 (40.5\%) previous ESWL, and 114 (17.0\%) PCNL. Renal anomalies were infrequent $(3.6 \%)$, and only 36 patients had a solitary functioning kidney.

\section{Stone Characteristics}

In total, 1969 stones were reported in the ureter and 942 stones in the renal system. The distribution of ureteric and renal stone location is shown in Figure 3. Ureteric stones were most often located in the distal ureter (40.8\%), and just over half of the renal stones (52.1\%) were in the lower pole. The median stone burden for ureteral stones was $47.1 \mathrm{~mm}^{3}$ (IQR 27.5 to 75.4 ) with a median length of $11.0 \mathrm{~mm}$ (IQR 7.0 to 12.0) and median width $6 \mathrm{~mm}$ (IQR 5.0 to 8.0). The renal stones in this cohort were larger with a median stone burden of 66.0 $\mathrm{mm}^{3}$ (IQR 28.4 to 117.8) with a median length of 10.0 $\mathrm{mm}$ (IQR 7.1 to 15.0 ) and median width $8.0 \mathrm{~mm}$ (IQR 5.0 to 10.0$)$.

\section{Intraoperative Details}

Of those patients given antibiotics, the majority (68.8\%) were administered a prophylactic dose, whereas $30.8 \%$ had a therapeutic course after the procedure.

Just over 20.6\% $(n=524)$ had a stent inserted before the definitive ureteroscopy procedure.

Reasons for these stent insertions included tight ureter (16.2\%) and ureteric narrowing/stenosis (17.2\%). Interestingly, the main indication for pre-stenting was the surgeons' preference (23.3\%). The median indwelling time in these patients was 30 days (IQR 20 to 60 days).
Turkey

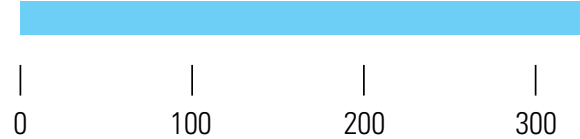

493

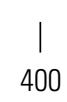

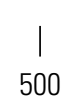

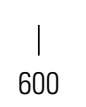


In $81.7 \%$ of procedures, a semi-rigid ureteroscope was used; the dimensions of semi-rigid ureteroscopes used ranged from $4.5 \mathrm{Fr}$ to $13 \mathrm{Fr}$, with the commonest being 6 to $8 \mathrm{Fr}$ as expected. In $30.8 \%$ a flexible ureteroscope was used, mainly fiber optic (66.2\%). The variety of instruments together with the other intraoperative details are shown in Table 2 and Figure 4.

In $27.9 \%$ of procedures a UAS was used. In almost half of these cases, the preferred UAS was sized 12 or $14 \mathrm{Fr}(47.7 \%)$. In the majority of cases $(76 \%)$, laser stone fragmentation was used, and in $24 \%$, pneumatic lithotripsy was used.

\section{Stent Details}

In the vast majority $(\mathrm{n}=2332 ; 91.3 \%)$ of the 2429 patients undergoing insertion of a ureteric drainage tube, a double J stent was used, rather than a ureteric stent. The reasons for stent insertion were concern about ureteric edema (54.9\%), lengthy procedure (4.9\%), difficult access (5.3\%), residual stones (23.6\%), use of a UAS (15.8\%), ureteric stricture $(12.6 \%)$ or lesion $(12.0 \%)$, solitary kidney (1.0\%), or surgical preference (29.8\%).

The majority were polyurethane $(85.1 \% \%)$ versus silicone $(6.1 \%)$ or other $(5.1 \%)$ materials.

Whereas 759 patients (31.2\%) had a multi-length stent, the remaining 1668 had a specific size; the commonest being $24 \mathrm{~cm}(18 \%), 26 \mathrm{~cm}(43.5 \%)$ or $28 \mathrm{~cm}(32.5 \%)$. The most commonly used double J stent diameters were $6 \mathrm{Fr}$ (54.5\%) and 4.7 Fr (34.9\%).

In terms of stent removal, with median duration being 27 days (IQR 15.0 to 37.0), the majority were undertaken in the operating room $(80.0 \%)$, with very few favoring self-removal by string (1.9\%) and a few opting for removal in the office setting (19.9\%). The operating room removals were mostly by rigid cystoscopy $(61.6 \%)$, followed by ureteroscopy (25.1\%) and flexible cystoscopy $(13.3 \%)$

\section{Discussion}

The morbidity associated with ureteric stenting is the aspect of stone treatment that patients tend to remember most vividly. Yet there remains a well-documented wide variation in practice. A recent systematic Cochrane review found it difficult to make firm conclusions about the practice because of what the authors described as moderate to low "certainty of evidence." Indeed, the review confirmed that there is no agreed-upon definition of an "uncomplicated" ureteroscopy. This being a global issue, we aimed to analyze this practice in an inter-continental study, with prospective data acquisition, and identify stenting patterns and preferences across the world[5]. This study allows some key conclusions, namely the types of stenting, duration of stent, antibiotic usage, and differences in management
TABLE 1.

Demographics

\begin{tabular}{|c|c|}
\hline Variable & $n(\%)$ \\
\hline Patients included & 2544 \\
\hline \multicolumn{2}{|l|}{ Gender } \\
\hline Male & $1635(64.3)$ \\
\hline Female & 904 (35.5) \\
\hline NA & $5(0.2)$ \\
\hline Age mean (range) & $49.0(18.0-93.0)$ \\
\hline BMI median (IOR) & $25.5(23.3-28.4)$ \\
\hline \multicolumn{2}{|l|}{ ASA } \\
\hline 1 & $1310(51.5)$ \\
\hline$\|$ & $1026(40.3)$ \\
\hline III & $188(7.4)$ \\
\hline IV & $11(0.4)$ \\
\hline V & $1(0.0)$ \\
\hline NA & $8(0.3)$ \\
\hline \multicolumn{2}{|l|}{ Medical history } \\
\hline$O A B$ & $31(1.2)$ \\
\hline UTI & $406(16.0)$ \\
\hline DM & $353(13.9)$ \\
\hline Chronic prednisolone use & $27(1.1)$ \\
\hline UPJ stenosis & $42(1.7)$ \\
\hline Longstanding immobilization & $17(0.7)$ \\
\hline \multicolumn{2}{|l|}{ Previous surgery } \\
\hline UPJ-plasty & $19(0.7)$ \\
\hline Stone treatment & $671(26.4)$ \\
\hline ESWL & $272(40.5)$ \\
\hline URS & $362(53.9)$ \\
\hline PCNL & $114(17.0)$ \\
\hline Other & $49(7.3)$ \\
\hline Ureter dilatation & $131(5.1)$ \\
\hline Other urological surgery & $113(4.4)$ \\
\hline Renal anomaly & $91(3.6)$ \\
\hline Ectopic kidney & $4(4.4)$ \\
\hline Horseshoe kidney & $10(11.0)$ \\
\hline Solitary kidney & $36(39.6)$ \\
\hline Other & $41(45.1)$ \\
\hline
\end{tabular}

ASA: American Society of Anesthesiologists; BMI: body mass index; DM: diabetes mellitus; ESWL: extracorporeal shockwave lithotripsy; NA: not available/specified; OAB: overactive bladder; OAC: oral anticoagulation; PCNL: percutaneous nephrolithotomy; UPJ: ureteropelvic junction; UTI: urinary tract infection; URS: ureteroscopy. 
TABLE 2.

Intraoperative details

\begin{tabular}{l|c} 
Variable & n (\%) \\
\hline Antibiotic regimen & $1749(68.8)$ \\
\hline Prophylactic & $784(30.8)$ \\
\hline Therapeutic & $524(20.6)$ \\
\hline Pre-stented & \\
\hline Reason for pre-stenting & \\
\hline \multicolumn{1}{|l}{ Surgeons' preference } & $122(23.3)$ \\
\hline Tight ureter & $85(16.2)$ \\
\hline Ureter stenosis & $90(17.2)$ \\
\hline Solitary kidney & $17(3.2)$ \\
\hline Other ${ }^{b}$ & $264(50.4)$ \\
\hline Indwelling time when pre-stented \\
(days), median (I0R) & $30.0(20.0-60.0)$ \\
\hline Ureter dilatation performed & $208(8.2)$ \\
\hline UAS & $710(27.9)$ \\
\hline $10 / 12$ & $226(31.8)$ \\
\hline $11 / 13$ & $85(12.0)$ \\
\hline $12 / 14$ & $339(47.7)$ \\
\hline Other & $60(8.5)$ \\
\hline
\end{tabular}

\begin{tabular}{lc}
\hline Type lithotripter & $1929(75.8)$ \\
\hline Laser & $608(4.3)$ \\
\hline Pneumatic & $25(0.1)$ \\
\hline Ballistic & $83(3.3)$ \\
\hline Other & $45.0(30.0-60.0)$ \\
\hline Duration surgery (min), median (IOR) & $924(36.3)$ \\
\hline Impacted stone & $13(0.5)$ \\
\hline Stone free & \\
\hline
\end{tabular}

UAS: ureteral access sheath, ${ }^{a}$ Multiple answers possible,

b Including obstructing stone.

for proximal stones. These findings are of relevance both for urologists and for those responsible for delivery of health care systems, and for the design of elective and emergency pathways.

With the benefit of a central online database, records were uploaded prospectively by the host centers in real time, and data entries could thus be audited both during and at the conclusion of the study period. Over 2500 records were thus collated, of which $10 \%$ were sampled for verification and validation of data entry. The global distribution is shown in Figure 2 and Appendix 1, highlighting the geographical spread of the centers contributing information to this study, with representation from Asia, the Middle East, Europe, and
Africa. This covers a range of health care systems, thus reducing any bias related to financial remuneration, for instance.

The high rate of ureteric stenting after ureteroscopy in this global cohort study $(2331 / 2540 ; 91.8 \%)$ is in line with previous reports, with the commonest justification being concern about ureteric edema leading to pain and/ or obstruction[6]. Although various guidelines have proposed that uncomplicated ureteroscopy can avoid routine stenting post-URS, there remains uncertainty about the zone that lies between "complicated" and "uncomplicated," and this is reflected in the high stenting rates worldwide[2,3,7-10]. The consistent high rates, regardless of country as per our study, suggest that this is a universal clinical concern and is unrelated to health care system. Nevertheless, almost $40 \%$ of the cases were for distal stones, and therefore one might expect a slightly lower propensity for stenting in these cases, compared with the $35 \%$ proximal stones for which a stent may be somewhat expected. It is possible to speculate that the reason for this high rate of stenting for distal stones is related to the concern about the higher rate of hospital readmission for unstented patients post-URS. However, this is not captured in the dataset as "surgical preference," which is listed as the reason in only $30.0 \%$ of cases. While there would undoubtedly be overlap with those reporting ureteric edema as the cause, one would expect "surgical preference" to be higher if listed.

The high proportion of stent removals that were undertaken in the operating room, as opposed to the office, was somewhat surprising, especially with the widespread availability of flexible cystoscopy and indeed a novel cystoscope with the grasper inbuilt[11]. Furthermore, the number of stents-on-string was surprisingly low, and this may be further developed, particularly following the coronavirus pandemic and the move to reducing unnecessary hospital visits. There remains uncertainty about the optimal timing for such stent removal-24 to 48 hours, $>72$ hours, etc-and this relates to the questions about duration of ureteric edema after ureteroscopy. Indeed, this is likely to be one of the factors that drives surgical preference for stenting. The pilot study by Paul et al., although it had a small sample size and retrospective design, suggested a higher proportion of postoperative issues for those with stent removal on day 3 versus on day 7 [12]. This study also found that almost $50 \%$ of patients preferred to avoid stent-on-string removal. Again, this is an area that warrants review through a validated patient reported outcome measure. Whether a local anesthetic office-based stent removal, or stent-on-string removal, it will be interesting to review whether the effects of the pandemic change the current global practice patterns. 
FIGURE 3.

Stone location

Ureter $\mathrm{n}=1969$ Renal $\mathrm{n}=942$

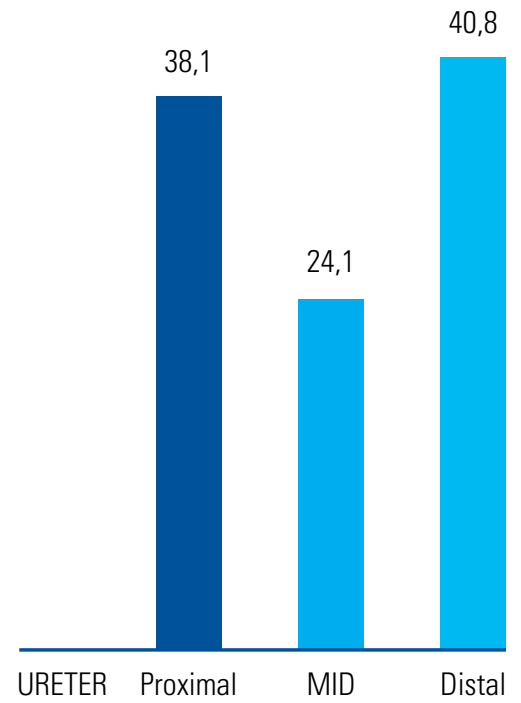

One of the key questions for ureteroscopic stone treatment is whether primary URS is feasible or whether a period of pre-stenting is required. This is likely to differ between the treatment of ureteric stones (in which the majority, though not all, of even proximal stones may be accessible at the initial procedure) and renal stones (in which safe practice, even with availability of access sheaths, may preclude reaching a stone in the case of a tight ureter). Our relatively low figure of $20.6 \%$ prestented patients is consistent with other reports: $11.9 \%$ preoperative placement for ureteric stones, $36.4 \%$ for renal stones[13]. This likely reflects the fact that most upper tracts are accessible by the miniaturization of the technology (with use of access sheaths and flexible ureterorenoscopes) but that some ureters will naturally remain too tight to permit safe retrograde passage without a period of passive dilation. This figure may also help with decision-making when the alternatives of ESWL and PCNL may be considered in terms of stonefree rates and choice of primary procedure. With the increasing recognition of mini-PCNL, the boundaries between URS and PCNL for renal stones have shifted over the past decade.

A further area of variability in practice is the prophylactic use of antibiotics and the prescription of antibiotics following the procedure. It remains fairly standard to use antibiotics at the time of URS, with approximately $2 / 3$ giving a dose at induction, though a relatively high proportion (almost $1 / 3$ ) continued a course of antibiotics after the procedure. While the latter practice would certainly be advisable when there may be a risk of ongoing infection (eg, recent

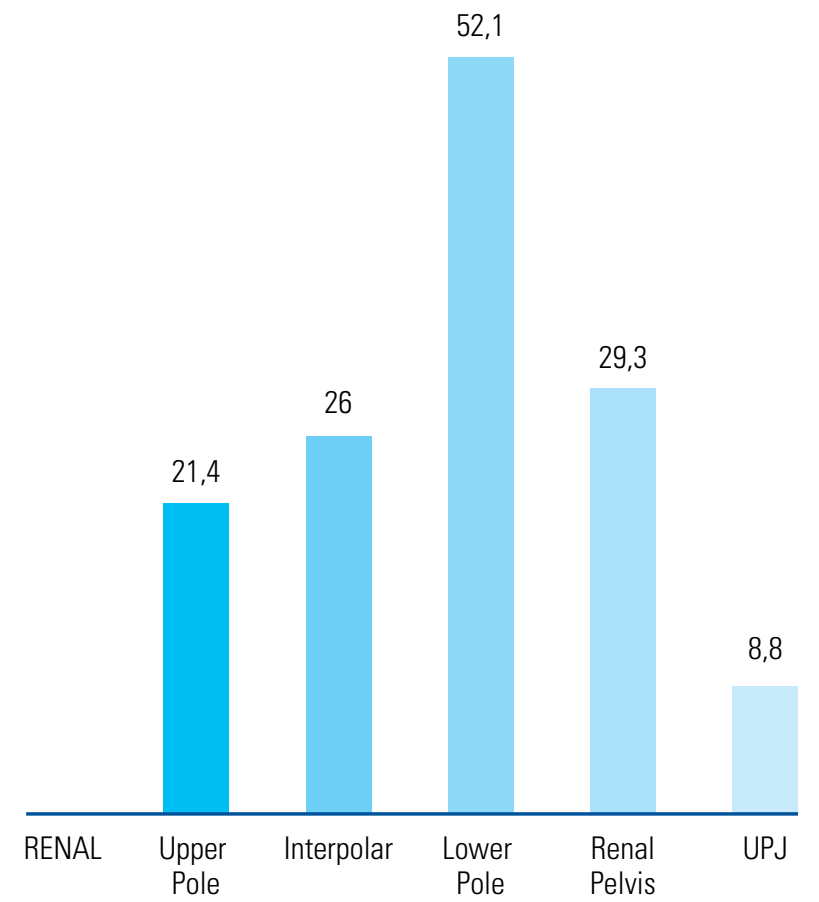

preoperative UTI, colonized stent, neuropathic patients, etc), the unquestionable global problem of antimicrobial resistance should lead to more judicious use of antibiotics. This should be reflected in guidelines, which have generally deferred to "local practice," with the lack of high-quality evidence being cited as a reason for the lack of a universal standard $[2,3,7,8]$. Of course, the other challenge is establishing the optimal type and dose of antibiotic (eg, an aminoglycoside, a penicillin, a cephalosporin, a quinolone, or a combination).

Urinary tract infections, hematuria, and stent-related symptoms are known postoperative problems associated with both the procedure as with stenting and may also be related to the duration of stenting. Whether reducing this stent indwell time (eg, by greater use of stent-onstring) would impact on the postoperative UTI rate, further research into optimal ureteric drainage would ideally incorporate such infection data.

In a global study such as this, one would expect to observe variations in the use of UAS; with 710 cases (27.9\%) using this, this figure is broadly as expected, especially for the number of proximal ureteric and renal stones. The proliferation of endourology expertise and technology would appear to have translated into use of this disposable. Although a higher rate would be expected in a renal stone rather than a ureteric stone study alone, the current figure illustrates familiarity with these sheaths globally; the relatively similar numbers for the 10/12 Fr size (31.8\%) and 12/14 Fr (47.7\%) may reflect local availability and experience. This may well also have an impact on stenting, as some who favor use of access 


\section{FIGURE 4}
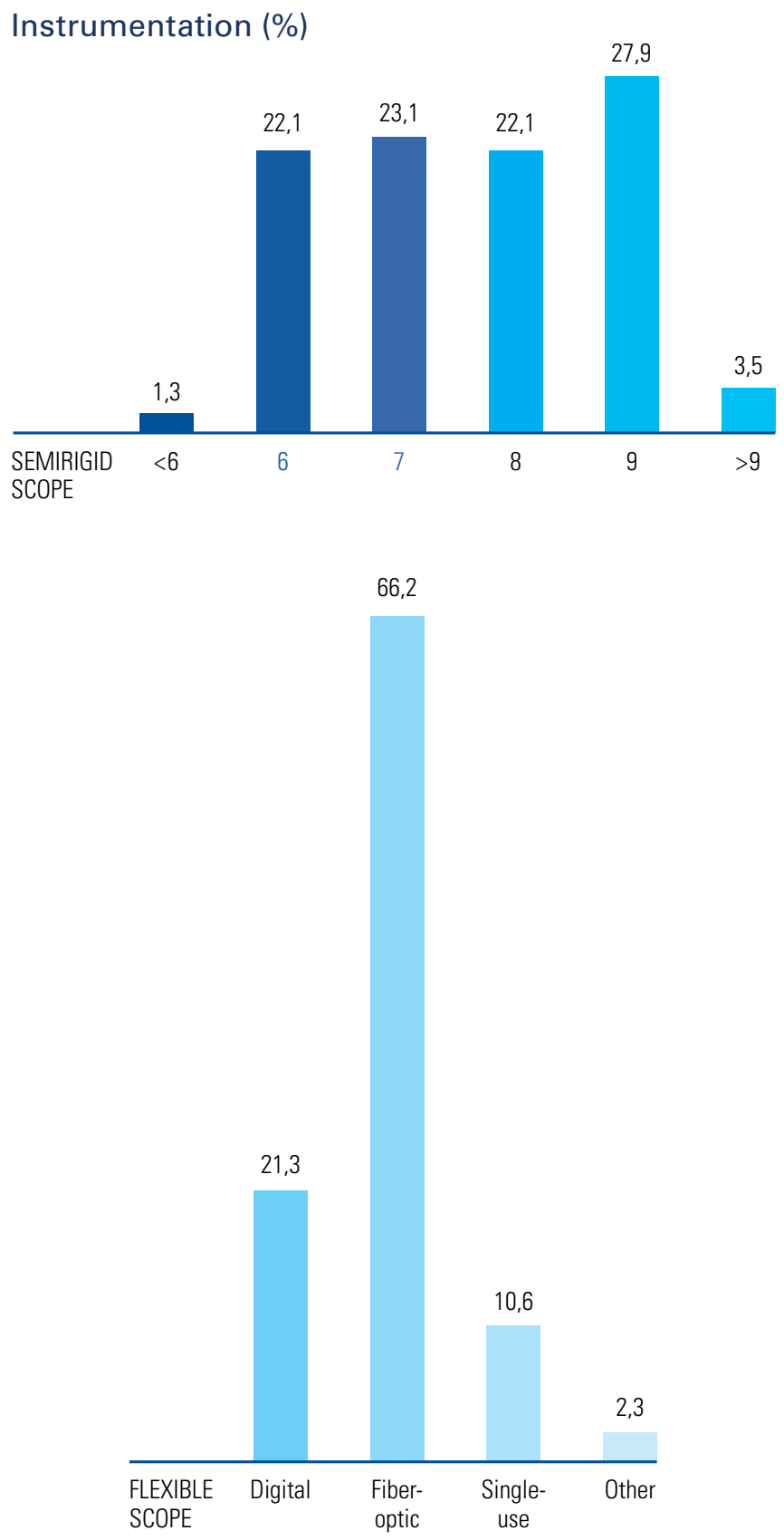

sheath may argue for the benefits of stenting due to theoretical issues about edema associated with UAS.

Limitations of this study include those of any study in which the data entry is by the individual center, but it has 2 advantages over other studies of practice (eg, online surveys, snapshot audits): (1) the centralized database was hosted by an independent organization, and (2) realtime entry meant that recall bias could be minimized, while the completion of datasets was maximized, and spurious or outlying data could be followed up. Data quality was assessed by an appointed audit committee, who were able to interrogate $10 \%$ of all cases, and confirmation of consent, imaging, and pathology reports were all accessed, and operative details were also verified. This process revealed that some centers kept original data in print form while others had moved to electronic data, and some used a combination.

While the questionnaire was designed in English, the consent process relied on translation locally, and the governance of informed consent relied on robust local Institutional Review Board approval at each center.

Finally, the listing of stent indication under "surgeon preference" was a broad variable without any qualitative description (ie, how this differed from concern about edema, residual fragments, etc).

\section{Conclusion}

This large multicenter cohort study reflects the current practice of stenting following ureteroscopy for stone disease and reveals widespread use of stenting despite an increase in suggestions that for certain cases, this morbid aspect of the surgery can be avoided. Where stents are used, the practice of stent-on-string remains underused, again reflecting current surgical preference. Future studies should address the indications for stenting in greater detail and help improve stent-related morbidity in the future

\section{Acknowledgments}

The authors would like to thank the following for their help.

Research Council Representatives: Rui Chen, Chinese Urological Association; Prodromos Philippou, Cyprus Urological Association; Yasser Farahat, Emirates Urological Society; Selcuk Guven, Eurasian Urooncological Association; Bernhard Ralla, German Society of Residents in Urology; Athanasios Papatsoris, Hellenic Urological Association; Lukman Hakim, Indonesian Urological Association;

Mohammad Hadi Radfar, Iranian Urological Association; Yoshihiko Tomita, Japanese Urological Association; Patrick Mburugu, Kenya Association of Urological Surgeons; Jae Young Park, Korean Urological Association; Teng Aik Ong, Malaysian Urological Association; Paul Villanti, Movember Foundation; Raed A. Azhar, Saudi Urological Association; André van der Merwe, South African Urological Association; YeongShiau Pu, Taiwan Urological Association.

Audit Committee members (for patient data audit): Yigit Akin, Samuel Yee.

SIU Central Office (for study coordination and database management): Christine Albino, Brittany Scarfo. 
This research study would not have been possible without the support from the Société Internationale d'Urologie.

We would like to thank the study team and all the research investigators who participated in the enrollment of patients for this study. See Appendix 1 for a complete list of participating centers.

\section{Author Contributions}

Ranan Dasgupta: drafting of the manuscript, acquisition of data, reviewing of the manuscript.

Teng Aik Ong, Jasmine Lim, Xiaofeng Gao, Lukman Hakim, Patrick Mburugu, Rohit Ajmera, Emrah Yuruk,

\section{References}

1. Raheem OA, Khandwala YS, Sur RL, Ghani KR, Denstedt JD. Burden of Urolithiasis: Trends in Prevalence, Treatments, and Costs. Eur Urol Focus. 2017;3(1):18-26. http://dx.doi.org/10.1016/j.euf.2017.04.001.

2. Türk C, Neisius A, Petřík A, Seitz C, Thomas K, Skolarikos A. EAU Guidelines on urolithiasis 2020. EAU Guidelines. 2020 Ed. 2020; presented.

3. Cloutier J, Anson K, Giusti G, Grasso M, Kamphuis G, Lahme S, et al. Update of the ICUD-SIU consultation on stone technology behind ureteroscopy. World J Urol. 2017;35(9):1353-9.

4. Seklehner S, Laudano MA, Jamzadeh A, Del Pizzo JJ, Chughtai $B$, Lee RK. Trends and inequalities in the surgical management of ureteric calculi in the USA. BJU Int. 2014 Mar;113(3):476-483. doi: 10.1111/bju.12372. Epub 2013 Nov 21. PMID: 24053734.

5. Ordonez M, Hwang EC, Borofsky M, Bakker CJ, Gandhi S, Dahm P. Ureteral stent versus no ureteral stent for ureteroscopy in the management of renal and ureteral calculi: a Cochrane review. Can Urol Assoc J. 2020;14(2):61-8.

6. De al Rosette J, Denstedt J, Geavlete P, Keeley F, Matsuda T, Pearle $M$, et al.; CROES URS Study Group. The clinical research office of the endourological society ureteroscopy global study: indications, complications, and outcomes in 11,885 patients. J Endourol. 2014;28(2):131-9. PMID: 24147820 D0I: 10.1089/end.2013.0436

7. Assimos D, Krambeck A, Miller NL, Monga M, Murad MH, Nelson $\mathrm{CP}$, et al. Surgical management of stones: American Urological Association/Endourological Society Guideline, PART I. J Urol. 2016;196(4):1153-60. http://dx.doi.org/10.1016/j.jur0.2016.05.090.
Yeong-Shiau Pu, Petrisor Geavlete, Raed A. Azhar, Shingai Mutambirwa: acquisition of data, critical revision of the manuscript for important intellectual content

Joyce Baard: study concept and design, critical revision of the manuscript for important intellectual content

Source of funding: This registry is funded by the Sociéte Internationale d'Urologie (SIU), 1155 Robert-Bourassa Blvd, Suite 1012, Montréal, Québec, Canada.

Ethics approval: Regional Medical Research Ethics Committee, Institutional Review Board (IRB) or a waiver for consent was obtained at each participating center prior to subject enrollment.

8. Assimos D, Krambeck A, Miller NL, Monga M, Murad MH, Nelson $C P$, et al. Surgical management of stones: American Urological Association/Endourological Society Guideline, PART II. J Urol. 2016;196(4):1161-9. http://dx.doi.org/10.1016/j.jur0.2016.05.091.

9. Excellence C. NICE Guideline - Renal and ureteric stones: assessment and management: NICE (2019) Renal and ureteric stones: assessment and management. BJU Int. 2019;123(2):220-232.

10. Haleblian G, Kijvikai K, De La Rosette J, Preminger G. Ureteral stenting and urinary stone management: a systematic review. J Urol. 2008;179(2):424-430.

11. Doizi S, Kamphuis G, Giusti G, Palmero JL, Patterson JM, Proietti S, et al. First clinical evaluation of a new single-use flexible cystoscope dedicated to double-J stent removal (IsirisTM): a European prospective multicenter study. World J Urol. 2017;35(8):1269-75.

12. Paul CJ, Brooks NA, Ghareeb GM, Tracy CR. Pilot study to determine optimal stent duration following ureteroscopy: three versus seven days. Curr Urol. 2018;11(2):97-102.

13. Assimos D, Crisci A, Culkin D, Xue W, Roelofs A, Duvdevani M, et al. Preoperative JJ stent placement in ureteric and renal stone treatment: Results from the Clinical Research Office of Endourological Society (CROES) ureteroscopy (URS) Global Study. BJU Int. 2016;117(4):648-654. 


\section{APPENDIX 1.}

Participating research centres

Institute

1. Shanghai Changhai Hospital, Second Military Medical University

2. Ningbo First Hospital, The Affiliated Hospital of Ningbo University

3. The First Affiliated Hospital of Guangzhou Medical University and G uangdong Key Laboratory of Urology

4. Renji Hospital, School of Medicine, Shanghai Jiao Tong University

5. Tongji Hospital, Tongji Medical College, Huazhong University of Science and Technology

6. Guangdong Second Provincial General Hospital, The Third Clinical Medical College of Southern Medical University.

7. Sismanoglio General Hospital

8. Aretaieion Academic Hospital

9. Aristotle University, 1st Department of Urology, Thessaloniki

10. University of Crete, Department of Urology

11. University Hospital of Larissa, Department of Urology

12. Jawahar Lal Nehru (JLN) Medical College

13. Kulkarni Reconstructive Urology Center

14. Sanglah Hospital, Department of Urology, Udayana University

15. Sardjito Hospital, Gadjah Mada University, Department of Urology, Jogjakarta

16. Cipto Mangunkusumo Hospital, Department of Urology, University of Indonesia

17. Dr. Soetomo Hospital, Department of Urology, Airlangga University

18. Hasan Sadikin Hospital, Department of Urology, Padjajaran University

19. Saiful Anwar Hospital, Department of Urology, Brawijaya University

20. Shahid Beheshti Hospital, Hamadan University of Medical Sciences

21. University of Basra - College of Medicine, Department of Urology

22. Pandya Memorial Hospital

23. University of Malaya Medical Centre

24. Selayang Hospital

25. Sarawak General Hospital
China

China

China

China

Wei Xue

China

Lei Cui

China

Guosheng Yang

Athanasios Papatsoris

Greece

Greece

Greece

Greece

Greece

India

India

Indonesia

Indonesia

Indonesia

Indonesia

Indonesia

Indonesia

Iran

Iraq

Kenya

Malaysia

Malaysia

Malaysia

\section{Athanasios Dellis}

Anastasios Anastasiadis

Charalampos Mamoulakis

Stavros Gravas

Rohit Ajmera

Sanjay Kulkarni

Kadek Budi Santosa

Indrawarman Soeroharjo

Nur Rasyid

Johan Renaldo

Safendra Siregar

Taufiq Nur Budaya

Seyed Habibollah Mousavibahar

Murtadha Almusafer

Sundeep Chavda

Teng Aik Ong

Rohan Malek

Guan Chou Teh 


\section{APPENDIX 1}

Participating research centres, Cont'd

Institute

26. Kuala Lumpur Hospital

27. Serdang Hospital

28. St. John Clinical Hospital of Emergency, Department of Urology

29. International Medical Center

30. King Abdulaziz University

31. Prince Sultan Military Medical City - Scientific Research Center

32. Sefako Makgatho Health Sciences University

33. SMG-SNU Boramae Medical Center

34. National Taiwan University Hospital, Department of Urology

35. En Chu Kong Hospital, Department of Urology

36. Cardinal Tien Hospital, Department of Urology

37. Kaohsiung Veterans General Hospital, Division of Urology, Department of Surgery

38. University of Health Sciences, Dept of Urology, Bagcilar Hospital

39. 9 Eylul University, Department of Urology

40. Gazi University School of Medicine, Department of Urology, Ankara

41. Baskent University, Faculty of Medicine, Department of Urology

42. Hacettepe University, Department of Urology, Ankara

43. Istanbul Medipol University, Department of Urology

44. Cerrahpasa School of Medicine, Istanbul University

45. Necmettin Erbakan University, Meram Medical School

46. University of Çukurova, Department of Urology, Adana

47. Konya Meram Education \& Research Hospital

48. Bulent Ecevit University, Department of Urology

49. Selcuk University Selcuklu Medical School

50. University of Minnesota, Department of Urology
Malaysia

Malaysia

Romania

Saudi Arabia

Saudi Arabia

Saudi Arabia

South Africa

South Korea

Taiwan

Taiwan

Taiwan

Taiwan

Turkey

Turkey

Turkey

Turkey

Turkey

Turkey

Turkey

Turkey

Turkey

Turkey

Turkey

Turkey

United States
Chia-Cheng Yu

Principal Investigator

Vijayan Manogran

Saiful Azli

Petrisor Geavlete

Raed A. Azhar

Raed A. Azhar

Ali Obied

Shingai Mutambirwa

Sung Yong Cho

Yeong-Shiau Pu

Chung-Cheng Wang

Hsu-Che Huang

mrah Yuruk

Guven Aslan

Bora Küpeli

M. Ilteris Tekin

Cenk Y. Bilen

Selcuk Guven

Bulent Onal

Mehmet Balasar

Ibrahim Atilla Aridogan

Mahmud Zahid Ünlü

N. Aydin Mungan

Mehmet Kaynar

Michael Borofsky 